\title{
A mental number line in human newborns
}

2

3

4

5

6 Rosa Rugani ${ }^{1, *}$, Marco Lunghi ${ }^{2, *}$, Elisa Di Giorgio ${ }^{2,3, *}$, Lucia Regolin ${ }^{1}$, Beatrice Dalla

7 Barba $^{4}$, Giorgio Vallortigara ${ }^{3}$, Francesca Simion ${ }^{2}$

8

9

$10{ }^{1}$ Department of General Psychology, University of Padova, Padova, Italy

$11{ }^{2}$ Department of Developmental and Social Psychology, University of Padova, Padova, Italy

$12{ }^{3}$ Center for Mind/Brain Sciences, University of Trento, Rovereto (Trento), Italy

$13{ }^{4}$ Women's and Children's Health Department, University of Padua

14 * These authors had equal contribution to the work.

18 Corresponding author:

19 Rosa Rugani, Department of General Psychology,

20 University of Padova, Via Venezia, 8, 35131, Padova, Italy

$21 \quad$ Fax +390498276600

22 E-mail address: rosa.rugani@unipd.it

23 Key words: number space association, human newborns, SNARC, mental number line, 24 numerical cognition, number sense. 


\section{ABSTRACT}

In the $19^{\text {th }}$ century Francis Galton first reported that humans represent numbers on a mental number line with smaller numbers on the left and larger numbers on the right. It has been suggested that this orientation emerges as a result of reading/writing habits for both words or numbers. Recent evidence in animals and infants in the first months of life has challenged the primary role of language in determining the left-to-right direction of spatialnumerical association, SNA. However, the possibility that SNA is learnt by early exposure to caregivers' directional biases is still open. Here we show that 55-hour-old newborns, once habituated to a number (i.e., 12), spontaneously associated a smaller number (i.e., 4) with the left side and a larger number (i.e., 36) with the right side of space. Moreover, SNA in neonates was not absolute but relative. The same number (i.e., 12) was associated with the left side whenever the previously experienced number was larger (i.e., 36), but with the right side whenever the number was smaller (i.e., 4). Control on continuous physical variables showed that the effect was specific of discrete magnitudes. Hence, soon after birth humans associate smaller numbers with the left space and larger numbers with the right space. These results constitute strong evidence that in our species SNA originates from pre-linguistic and biologically precursors in the brain.

0

1

\section{SIGNIFICANCE STATEMENT}

For human adults, the representation of number and space is profoundly intertwined. Humans represent numbers on a left to right oriented Mental Number Line (MNL), with small numbers located on the left and larger ones on the right. How do these connections 
1 arise? Do we learn to associate numbers with space throughout cultural learning and social interactions or is this association rooted in the biology of the human brain? We showed that neonates spontaneously associate numbers with space. After being habituated to a certain number, neonates associated a smaller number with the left and a larger number with the right side. This evidence demonstrates that a predisposition to map numbers onto space is rooted in human neural systems.

Non-symbolic numerical skills are widespread in the animal kingdom (1). Pre-verbal infants

9 (2,3) and non-human species (4) can extrapolate numerical magnitude from an array of elements,

10 showing a non-symbolic number comprehension $(5,6)$. In humans, this comprehension is present

11 early in infancy (2) and can be assessed in adults whenever the use of language is prevented $(7,8)$.

12 Non-symbolic numerical tasks are easier, as the difference between the numbers increases (distance 13 effect) and harder as the numerical magnitude increases (size effect), for both humans (9) and 14 animals $(8,10)$. These similarities are suggestive of a shared, ancient, non-verbal numerical 15 mechanism (8). Therefore, uniquely human mathematical abilities seem to be based on a 16 developmental and evolutionarily ancient "number sense" (11).

18 bers along a left-right oriented continuum (12). Adults are faster at processing small numbers when 19 responses are executed on the left side of space and faster for large numbers when responses are ex20 ecuted on the right side of space (spatial-numerical association of response codes, SNARC effect) 21 (13). Several studies suggested that the left-to-right orientation of the mental number line is an out22 come of exposure to formal instruction and that the mapping of number onto space would be a by23 product of culture, based on reading/writing conventions and tool use, such as rulers (14). People 24 for whom Arabic is the main language show an inverted SNARC effect (15), whereas people with 25 mixed reading habits (i.e. those brought up reading both left-to-right and right-to-left) show no 26 SNARC effect at all (16). However, an increasing number of studies support a phylogenetic origin 
1 of the mental number line. Seven-month-old infants looked longer at increasing (e.g. 1-2-3) but not

2 at decreasing (e.g. 3-2-1) magnitudes displayed in a left-to-right spatial orientation (17). Eight-

3 month-old infants oriented their attention toward the left after having seen a small number (i.e., 2),

4 and toward the right after having seen a large number (i.e., 9) (18). Infant evidence excludes a pri-

5 mary influence of verbal counting in SNA orientation. However, this could be determined by the in-

6 teractions with adults and the external world (19). A tendency to look longer at numerousness from

7 left-to-right has been reported in our species $(3,17)$. However, this is only a partial evidence of the

8 SNA, because increasing the looking time from right-to-left has not been reported for decreasing

9 sequences.

Adult Clark's nutcrackers (20) and rhesus monkeys (21) have shown unilateral, left-to-right oriented bias to associate numerousness with space. Nevertheless, these biases could depend on continuous extents, which were not systematically controlled for. A complete evidence of a nonverbal SNARC-like phenomenon has been provided, up to now, only in domestic chicks, which

14 preferentially respond to small numbers on the left side and to large numbers on the right side of 15 space (22). Chicks associated a same non-symbolic number (i.e., an array of 8 squares) either with 16 the left side, in the 8-32 range, or with the right side, in the 2-8 range. Such "relativity" of SNA is a 17 fundamental characteristic of the human mental number line. one that drives the effect in humans (16). Birds have laterally placed eyes, virtually complete nerve crossings at the optic chiasm and minimal interhemispheric connections, giving rise to a strong lat-

21 eralization of function in everyday behavior (23). Humans, in contrast, like other primates, have 22 frontally placed eyes, only partial crossing of nerves at the optic chiasm and strong interhemispheric connectivity. As a result, they show visual lateralization only in restricted conditions of vision (e.g. lateral presentation of briefly-presented stimuli) (24). The only way to discover the root of the human mental number line (20) is exploring whether human newborns, under minimal or no exposure to adults' scanning biases, manifest SNA. 


\section{Results}

6 hour-old ( $\mathrm{SD}=28.16$, range: $12-117 \mathrm{~h})$, took part at the Experiment 1 and were tested with an in-

7 fant-control habituation technique.

8 Habituation and test stimuli consisted of static two-dimensional images. All stimuli

9 contained a well-defined number of black square elements, average luminance $0.4 \mathrm{~cd} / \mathrm{m}^{2}$, depicted

10 on identical white square area of the dimension of $17.5 \mathrm{~cm} \mathrm{x} 17.5 \mathrm{~cm}(694.68$ pixels $\times 694.68$

11 pixels), subtending a visual angle of $30.3^{\circ} \times 30.3^{\circ}$ (average luminance $103 \mathrm{~cd} / \mathrm{m}^{2}$ ). The number, the

12 dimension and the position of the elements varied as a function of the experimental conditions. The

13 distance elapsing between the closer edge of each stimulus and the center of the screen was of 4.25

$14 \mathrm{~cm}\left(8.06^{\circ}\right)$

15 For the habituation phase we used five stimuli depicting 12 elements with a different spatial disposi-

16 tion. Each stimulus lasted $500 \mathrm{~ms}$ without any interval among stimuli presentation. We decided to

17 employ five stimuli during the habituation phase in order to i) attract and maintain newborns' atten-

18 tion and ii) prevent the newborns from learning to identify the stimuli on the basis of the spatial

19 disposition of the black squares.

After the habituation phase with the number 12, a sequence of two different trials,

21 counterbalanced between participants, was administered: half of the newborns had the small

22 number in the first test trial and the large number in the second one (4-36), whereas the other half

23 had the large number in the first test trial and the small number in the second one (36-4). In each

24 test trial the same stimulus was simultaneously presented on the right and on the left side of a

25 monitor. Each black square element measured $1.1 \mathrm{~cm}$ x $1.1 \mathrm{~cm}$ (43.67 pixels x 43.67 pixels), 
1 subtending a visual angle of $2.1^{\circ} \times 2.1^{\circ}$. Stimuli employed in the habituation phase were arrays

2 composed of 12 black square elements. Test stimuli comprised a number of elements either smaller

3 (4 black squares, for the small number test trial) or larger (36 black squares, for the large number

4 test trial) than the number experienced during habituation (i.e., 12). In the present study, for the

5 convenience of explanation, we calculated the percentage index using the stimulus on the left side

6 for all experimental conditions. Therefore, scores significantly below 50\% indicate a visual

7 preference for the stimuli on the right side of the screen whereas, scores significantly above $50 \%$

8 indicate a preference for the stimulus on the left side.

9 All newborns included in the final sample reached the habituation criterion and they didn't

10 show any spatial biases, $t_{23}=.56, p=0.582\left(\mathrm{M}_{\text {left }}=52.25 \%\right.$, see Fig. 1$)$.

11 We carried out a repeated measure ANOVA with Test Trial Order (4-36 and 36-4) as a

12 between-participants factor and Stimulus (4vs.4 and 36vs.36) as within-participants factor on the

13 percentage of total fixation time toward left stimuli. The analysis revealed a significant main effect

14 of Stimulus, $F_{1,22}=14.29, p<0.001, \eta_{p}^{2}=0.48$ (number $4, \mathrm{M}_{\mathrm{left}}=64.04 \%, \mathrm{SD}=18.65, t_{23}=3.69, p$

$15=0.001 ;$ number $\left.36, \mathrm{M}_{\mathrm{left}}=36.08 \%, \mathrm{SD}=22.85, t_{23}=-2.98, p=0.007\right)$. Regardless of the Test Trial

16 Order, newborns looked longer at the left-stimulus in the $4 v s .4$ trial, and at the right-stimulus in the

$1736 v s .36$ trial. However, since the first test trial could, theoretically, influence the second one, we

18 analyzed only the first test trial. Results confirm that when the two stimuli depicted a number

19 smaller than 12 (4vs.4, Fig.1), newborns looked longer at the left-stimulus $\left(\mathrm{M}_{\text {left }}=61.25 \%, \mathrm{SD}=\right.$

$\left.2017.44, t_{11}=2.24, p=0.047\right)$, when the stimuli depicted a number larger than 12 (36vs.36, Fig.1),

21 they looked longer at the right-stimulus $\left(\mathrm{M}_{\mathrm{left}}=28.00 \%, \mathrm{SD}=24.68, t_{11}=-3.09, p=0.010\right)$.

These data suggest that at birth the association between small numerousness with the left

23 side of space and large numerousness with the right side of space is already present. However, since

24 the squares were identical in size, newborns' preferences could have been driven by numerical or by

25 continuous physical variables (overall perimeter and overall area). 
Experiment 2: The SNARC effect exhibits "relativity" in human newborns. In

2 Experiment $2 \mathrm{a}$ and $2 \mathrm{~b}$, we tested for two fundamental characteristics of the SNA in newborns: i) its

3 independence from continuous physical variables; ii) its relative nature.

4 To exclude any possible use of continuous physical variables, we used squares of different

5 dimensions during habituation and test trials. By controlling for the overall perimeter (the

6 summation of perimeters of all squares depicted in both habituation and test stimuli was identical)

7 we also controlled for the overall area (if the overall perimeter of two arrays of two-dimensional

8 squares is identical, a negative correlation exists between numbers and overall area) (6).

9 To test SNA's relativity, we habituated 12 neonates (Mean $=64.66 \mathrm{~h}, \mathrm{SD}=29.74$, range $29-$

$10126 \mathrm{~h}$; Experiment 2a) with the number 4 and a second group of 12 newborns (Mean $=52.75 \mathrm{~h}$, SD

$11=42$, range $11-135 \mathrm{~h}$, Experiment $2 \mathrm{~b}$ ) with the number 36. During a single test trial, both groups

12 were presented with two identical stimuli, each depicting 12 squares, one on the left and one on the

13 right side of the monitor.

14 In Experiment $2 \mathrm{a}$ and $2 \mathrm{~b}$, we equated the overall perimeter (i.e. summation of the perimeter

15 of all black squares) and the overall area (i.e. summation of the area of all black squares) of the

16 stimuli presented in the habituation and in the test trials. As a consequence, we obtained a negative

17 correlation between the overall number of elements and their overall area.

As in Experiment 1, we used five stimuli with a different spatial disposition of the elements

19 during the habituation phase. Specifically, in Experiment 2a, for the habituation phase we employed

20 stimuli comprising of 4 black squares of $3.3 \mathrm{~cm} \times 3.3 \mathrm{~cm}$ (131.50 pixels x 131.50 pixels),

21 subtending a visual angle of $6.3^{\circ} \times 6.3^{\circ}$. The overall perimeter of the 4-elements was $58.2 \mathrm{~cm}$. Test

22 trial stimuli were 12 static black squares of $1.1 \mathrm{~cm} \mathrm{x} 1.1 \mathrm{~cm}$ and therefore, with an overall perimeter

23 of $58.2 \mathrm{~cm}$. Importantly, the overall area of the 4-element stimuli $\left(43.6 \mathrm{~cm}^{2}\right)$ was larger than that of

24 the 12-element stimuli $\left(14.5 \mathrm{~cm}^{2}\right)$. If the overall area, when the overall perimeter of the stimuli is

25 identical, were the crucial factor underlying space-number association, newborns would have

26 looked longer at the stimulus on the right side. 
As in Experiment 2a, in Experiment $2 \mathrm{~b}$ the overall perimeter between the habituation stimuli and the test stimuli was identical $(158.4 \mathrm{~cm})$. Habituation stimuli were 36 black squares $(1.1 \mathrm{~cm} \mathrm{x}$ $1.1 \mathrm{~cm}$ ), whereas test stimuli were 12 static black squares, measuring $3.3 \mathrm{~cm} \times 3.3 \mathrm{~cm}$. The overall area of the 36-elements stimuli $\left(43.6 \mathrm{~cm}^{2}\right)$ was smaller than that of the 12-elements stimuli $(130.7$ $\mathrm{cm}^{2}$ ). If the overall area, when the overall perimeter of the stimuli is identical, were the crucial factor underlying space-number association, newborns would have looked longer at the stimulus on the left side.

All newborns included in the final sample reached the habituation criterion and they didn't show any spatial biases either in Experiment $2 \mathrm{a}, t_{11}=.73, p=0.480\left(\mathrm{M}_{\mathrm{left}}=54.25 \%\right.$, see Fig.2 $)$ and in Experiment 2b, $t_{11}=.02, p=0.980\left(\mathrm{M}_{\mathrm{left}}=50.17 \%\right.$, see Fig. 3$)$.

We ran a univariate ANOVA with Experiment ( $2 \mathrm{a}$ and $2 \mathrm{~b}$ ) as a between-participants factor on the percentage of total fixation time toward left stimuli. The analysis revealed a significant main effect of Experiment, $F_{1,22}=19.671, p=0.001, \eta_{p}^{2}=0.472$. Neonates habituated with number 4 range. 
1 be accounted for either by innate or learning mechanisms. Up to now a complete association

2 between small numbers and left space, and large numbers and right space has been provided solely

3 in three-day-old domestic chicks (22). This evidence in completely inexperienced birds suggests

4 that the role of reading and writing directionality is secondary in determining the orientation of the

5 SNA $(41,21)$

6 Caution has been urged, however, when using animal models to understand the origin of the

7 orientation of human MNL (42). Convergent evolution, in which species from diverse evolutionary

8 lineage could independently develop similar features (43), and strong differences in brain

9 organization and lateralization $(21,44)$ could affect interpretation of comparative evidence (but see

$1045,46)$. Nevertheless, comparative as well as developmental studies have been, so far, unable to

11 unequivocally address the origin of human MNL. We overcame these limits by studying, for the

12 very first time, human newborns with a very limited visual experience.

Here we provide evidence for a complete, relative and magnitude-based SNA in neonates.

14 Hour-old newborns, initially habituated with a certain numerical value, spontaneously associated a

15 smaller number with the left space and a larger number with the right space (Experiment 1). This

16 association did not depend on the absolute magnitude of the number itself. Newborns habituated

17 with number 4 associated the number 12 with the right (Experiment 2a), while newborns habituated

18 with number 36 associated the number 12 with the left (Experiment 2b). This shows that SNA in

19 newborns exhibits relativity.

20 Moreover, these findings cannot be explained by continuous physical variables. In fact, in

21 Experiment 2a and in Experiment 2b, we controlled for the overall perimeter, obtaining an inverse

22 correlation between overall area and number. Had newborns associated space to overall area,

23 instead of number, their choices would have been the opposite to what reported. 
1 The fact that day-old newborns rely on numerical rather than on quantitative information is in line

2 with previous research which highlighted that, at the start of postnatal experience, we spontaneously

3 use abstract numerical cues (3). Number is considered a fundamental perceptual feature that our

4 brain process early to attain a complete representation of the external world $(47,48,49,50,51,52$,

5 53; for a different perspective see 54). It seems that numerical competence did not emerge de novo

6 in linguistic/symbolic adult humans, but was likely built on precursors available soon after birth

7 (11, 55, 56). From this perspective, a non-symbolic number sense can be considered a

8 developmental building block for the uniquely human capacity for mathematics (57). In support of

9 this idea, it has been found that impairments to the non-symbolic numerical system are related to

10 the occurrence of dyscalculia (58). The acuity of the non-symbolic numerical system is predictive

11 of mathematical ability in early childhood (59) and throughout training it improves proficiency in

12 symbolic mathematics (60). Our data strengthen the range of influence of non-symbolic numerical

13 system on the symbolic one, showing that this affects also the directionality of the MNL.

14 Overall our findings show that SNA occurs with minimal experience, supporting the 15 biological origin of SNA. This did not exclude that verbal $(16)$ and non-verbal $(19,25)$ experiences

16 can modulate its directionality. Even if the orientation of the MNL reflects cultural effects (16), its

17 widespread presence across diverse cultures supports the idea that the association between number

18 and space is a universal cognitive strategy (61). Our evidence is strong, but also challenging. It is a

19 starting point to disentangle the relative role and weight of cultural and biological factors in

20 determining the orientation of the human mental number line. 


\section{Acknowledgements}

2 The authors are deeply indebted to Professors G. Perilongo, head of the Department of

3 Health of woman and child and E. Baraldi, head of the Intensive Care and neonatal Pathology unit

4 of the University of Padova. . We would also like to thank the nursing staff, the babies and their

5 parents for their collaboration; Elena Berto, Daniela Carà and Silvia Dalò for assistance with new-

6 born testing. A special thank to Kim Anne Barchi for the help during the editing of the manuscript.

7 This study was carried out within the framework of the agreement between the University

8 and the Azienda Ospedaliera of Padova prot.: 91644645, and was supported by 'Progetto di Ateneo'

92012 to L.R. prot. CPDA127200) and by ERC Advanced Grant to G.V. (PREMESOR ERC-2011-

10 ADG_20110406).

\section{Author Contributions}

R.R., M.L., E.D.G., L.R. and F.S. designed the study; R.R., M.L. and E.D.G. created the

14 stimuli; M.L. and E.D.G. collected, analyzed the data and did the statistical analyses; R.R., M.L.,

15 E.D.G., L.R., G.V. and F.S. interpreted the data, discussed the results; R.R., M.L. and E.D.G wrote

16 the manuscript; L.R., G.V., B.D.B. and F.S. critically revised the manuscript.

\section{Author Information}

19 The authors declare no competing financial interests.

20 Correspondence and requests for materials should be addressed to R.R. (rosa.rugani@unipd.it). 


\section{References}

2 1. Vallortigara G (2014) Foundations of number and space representations in non-human species. In

3 Evolutionary Origins and Early Development of Number Processing, eds Geary D-C, Bearch D-B

4 \& Mann Koepke K (Elsevier, New York), pp 35-66.

5 2. Cordes S, Brannon E-M (2009) Crossing the divide: Infants discriminate small from large 6 numerosities. Dev Psychol 45(6):1583-1594.

7 3. Izard V, Sann C, Spelke E-S, Streri A (2009) Newborn infants perceive abstract numbers. Proc.

$8 \quad$ Natl Acad Sci USA 106 (25):10382-10385.

9 4. Vallortigara G (2012) Core knowledge of object, number, and geometry: a comparative and 10 neural approach. Cogn Neuropsychol 29 (1-2):213-236.

11 5. Feigenson L, Dehaene S, Spelke E-S (2004) Core systems of number. Trends Cogn Sci 8(7):30712314.

13 6. Rugani R, Castiello U, Priftis K, Spoto A, Sartori L (2017) What is a number? The interplay

14 between number and continuous magnitudes. Behav Brain Res in press.

15 7. Cordes S, Gelman R, Gallistel C-R, Whalen J (2001) Variability signatures distinguish verbal

16 from nonverbal counting for both large and small numbers. Psychon Bull Rev 8(4):698-707.

17 8. Cantlon J-F, Brannon E-M (2007) Basic Math in Monkeys and College Students. PLoS Biol 5, $18 \mathrm{e} 328$.

19 9. Moyer R-S, Landaeur T-K (1967) Time required for judgments of numerical inequality. Nature $20 \quad 215: 1519-1520$.

21 10. Scarf D, Hayne H, Colombo M (2011) Pigeons on Par with Primates in Numerical Competence. 22 Science 334:1664.

23 11. Dehaene S. (2011) The number sense: How the mind creates mathematics, revised and updated 24 edition (New York, Oxford University Press).

25 12. Galton F (1880) Visualised numerals. Nature 21:252-256. 
1 13. Dehaene S, Bossini S, Giraux P (1993) The mental representation of parity and number

2 magnitude. J Exp Psychol Gen 122:371-396.

3 14. Rugani R, de Hevia M-D (2017) Number-space associations without language: Evidence from

4 preverbal human infants and non-human animal species. Psychon Bull Rev 24:352-369.

5 15. Zebian S. (2005) Linkages between number concepts, spatial thinking and directionality of

6 writing: The SNARC effect and the REVERSE SNARC effect in English and in Arabic

7 monoliterates, biliterates and illiterate Arabic speakers. J Cog Cult 5:165-190.

8 16. Shaki S, Fischer M-H, Petrusic W-M (2009) Reading habits for both words and numbers

9 contribute to the SNARC effect. Psychon Bull Rev 16:328-331.

10 17. de Hevia M-D, Izard V, Coubart A, Spelke E-S, Streri A (2014) Representations of space, time,

11 and number in neonates. Proc Natl Acad Sci USA 111(13): 4809-4813.

12 18. Bulf H, de Hevia M-D, Macchi-Cassia V (2015) Small on the left, large on the right: Numbers

13 orient preverbal infants' visual attention onto space. Dev Sci 19(3):394-401.

14 19. Patro K, Fischer U, Nuerk H-K, Cress U (2016) How to rapidly construct a spatial-numerical

15 representation in preliterate children (at least temporarily). Dev Sci 19(1):126-144.

16 20. Rugani R, Kelly D-M, Szelest I, Regolin L, Vallortigara G (2010) Is it only humans that count

17 from left to right? Biol Lett 6(3):290-292.

18 21. Drucker C-B, Brannon E-M (2014) Rhesus monkeys (Macaca mulatta) map number onto space.

19 Cognition 132:57-67.

20 22. Rugani R, Vallortigara G, Priftis K, Regolin L (2015) Number-space mapping in the newborn

21 chick resembles humans' mental number line. Science 347(6221):534-536.

22 23. Vallortigara G, Versace E (2017) Laterality at the neural, cognitive, and behavioral levels. In

23 APA Handbook of Comparative Psychology: Vol. 1. Basic Concepts, Methods, Neural Substrate,

24 and Behavior, eds Call J (American Psychological Association, Washington DC), pp 557-577. 
1 24. Ocklenburg S (2017) Tachistoscopic Viewing and Dichotic Listening. In Lateralized Brain

2 Functions, eds Rogers L-J, Vallortigara G (Springer Verlag, New York), pp 3-28.

3 25. Bächtold D, Baumüller M, Brugger P. (1998) Stimulus-response compatibility in

4 representational space. Neuropsychologia 36:731-735.

5 26. Harvey B-M, Klein B-P, Petridou N, Dumoulin S-O (2013) Topographic Representation of

6 Numerosity in the Human Parietal Cortex. Science 341(6150):1123-1126.

7 27. Ditz H-M, Nieder A (2015) Neurons selective to the number of visual items in the corvid

8 songbird endbrain. Proc Natl Acad Sci USA 112(25):7827-7832.

9 28. Prechtl H, O'Brien M-J (1982) Behavioral states of the full term newborn: The emergence of a

10 concept. In Psychobiology of the human newborn eds Stratton P. (New York, Wiley), pp 53-73

11 29. Horowitz F-D, Paden L, Bhama K, Self P (1972) An infant-control procedure for studying infant

12 visual fixation. Dev Psychol 7(90).

13 30. Sokolov E-N (1963) Perception and the condition reflex (New York, Macmillan).

14 31. Slater A, Earle D-C, Morison V, Rose D (1985) Pattern preferences at birth and their interaction

15 with habituation-induced novelty preferences. J Exp Child Psychol 39:37-54.

16 32. Abramov I, et al (1982) The retina of the newborn human infant. Science 217:265-267.

17 33. Atkinson J, Braddick O (1989) Development of basic visual functions. In Infant development

18 eds Slater A, Bremner G (London, Erlbaum), pp 7-41.

19 34. Valenza E, Simion F, Macchi-Cassia V, Umiltà C (1996) Face preference at birth. J Exp Psychol

20 Human 22:892-903.

21 35. Di Giorgio E, Leo I, Pascalis O, Simion F (2012) Is the face-perception system human-specific 22 at birth? Dev Psychol 48:1083-1090. 
1 36. Cohen L-B (1972) Attention-getting and attention-holding processes of infant visual prefer-

2 ences. Child Dev 43:869-879.

3 37. Lourenco S-F, Longo M-R (2010) General magnitude representation in human infants. Psychol $4 \quad$ Sci 21(6):873-881.

5 38. Adachi I (2014). Spontaneous spatial mapping of learned sequence in chimpanzees: Evidence

6 for a SNARC-like effect. PLoS One 9: e90373.

7 39. Rugani R, Rosa-Salva O, Regolin L (2014) Lateralized mechanisms for encoding of object. Be-

8 havioral evidence from an animal model: the domestic chick (Gallus gallus). Front Psychol 5.

9 40. Rugani R, Vallortigara G, Regolin L (2016) Mapping number to space in the two hemispheres of 10 the avian brain. Neurobiol Learn Mem 133:13-18.

11 41. Brugger P (2015) Chicks with a number sense. Science, 347(6221): 477-478.

12 42. Patro K, Fischer U, Nuerk H-K, Cress U (2016) How to rapidly construct a spatial-numerical 13 representation in preliterate children (at least temporarily). Dev Sci 19(1): 126-144.

14 43. Emery N-J, Clayton N-S (2004) The mentality of crows: convergent evolution of intelligence in 15 corvids and apes. Science, 306(5703):1903-1907.

16 44. Fischer M-H, Shaki S (2016) Measuring spatial-numerical associations: evidence for a purely

17 conceptual link. Psychol Res 80(1):109-112.

18 45. Rugani R, Vallortigara G, Priftis K Regolin L (2015) Response to Comments on ”Number-space

19 mapping in the newborn chick resembles humans' mental number line". Science 348:1438.

20 46. Rugani R, Vallortigara G, Priftis K Regolin L (2016) Response: "Newborn chicks need no num-

21 ber tricks. Commentary: Number-space mapping in the newborn chickresembles humans' mental 22 number line". Front Hum Neurosci 10:31. 
1 47. Burr D, Ross J (2008) A visual sense of number. Curr Biol 18(6):425-428.

2 48. DeWind N-K, Adams G-K, Platt M-L, Brannon E-M (2015) Modeling the approximate number

3 system to quantify the contribution of visual stimulus features. Cognition, 142:247-265.

4 49. Anobile G, Cicchini G-M, Burr DC (2016) Number as a primary perceptual attribute: a review. 5 Perception $45(1-2), 5-31$.

6 50. Cicchini G-M, Anobile G, Burr D-C (2016) Spontaneous perception of numerosity in humans.

$7 \quad$ Nature Commun, 7: 12536.

8 51. Fornaciai M, Cicchini G-M, Burr D-C (2016) Adaptation to number operates on perceived ra-

9 ther than physical numerosity. Cognition 151: 63-67.

10 52. Fornaciai M, Brannon E-M, Woldorff M-G, Park J (2017) Numerosity processing in early visual 11 cortex. NeuroImage 157:429-438.

12 53. Park J, DeWind N-K, Woldorff M-G, Brannon E-M, (2016) Rapid and direct encoding of 13 numerosity in the visual stream. Cereb Cortex 26: 748-763.

14 54. Leibovich T, Ansari D (2016) The symbol-grounding problem in numerical cognition: A review 15 of theory, evidence, and outstanding questions. Can J Exp Psychol 70(1):12.

16 55. Carey S (2009) The origin of concepts (Oxford University Press).

17 56. Vallortigara G (2017) An animal's sense of number. In The nature and development of mathe-

18 matics. Cross disciplinary perspective on cognition, learning and culture, eds Adams J-W, Barmby

19 P, Mesoudi A (Routledge, New York), pp 43-65.

20 57. Spelke E-S (2000). Core knowledge. Am Psychol 55: 1233-1243.

21 58. Wilson A-J, Dehaene S (2007) Number sense and developmental dyscalculia. Hum Behav Learn

22 Dev Brain: Atypical Dev 2: 212-237. 
1 59. Starr A, Libertus M-E, Brannon E-M (2013) Infants show ratio-dependent number discrimina-

2 tion regardless of set size. Infancy 18: 927-941.

3 60. Park J, Brannon E-M (2013) Training the approximate number system improves math proficien-

4 cy. Psychol Sci 24: 2013-2019.

5 61. Göbel, S-M, Shaki S, Fischer M-H (2011) The cultural number line: A review of cultural and

6 linguistic influences on the development of number processing. J Cross-Cultural Psychol 42: 543-

7565.

8 


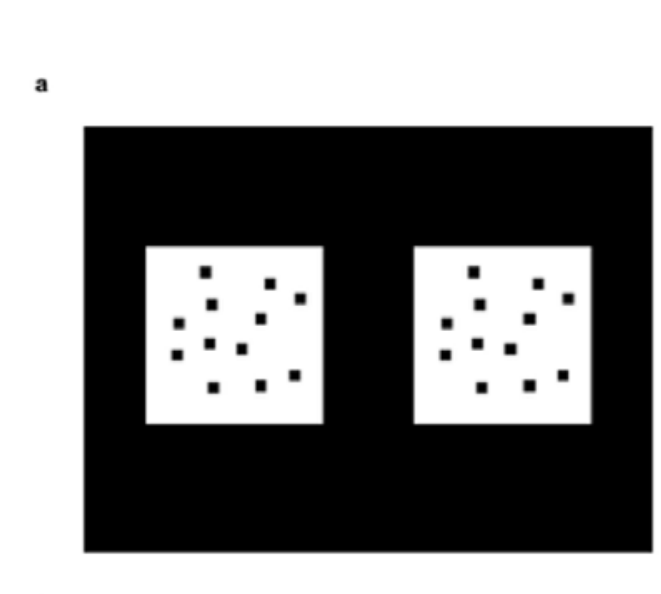

Experiment 1

c

Habituation phase
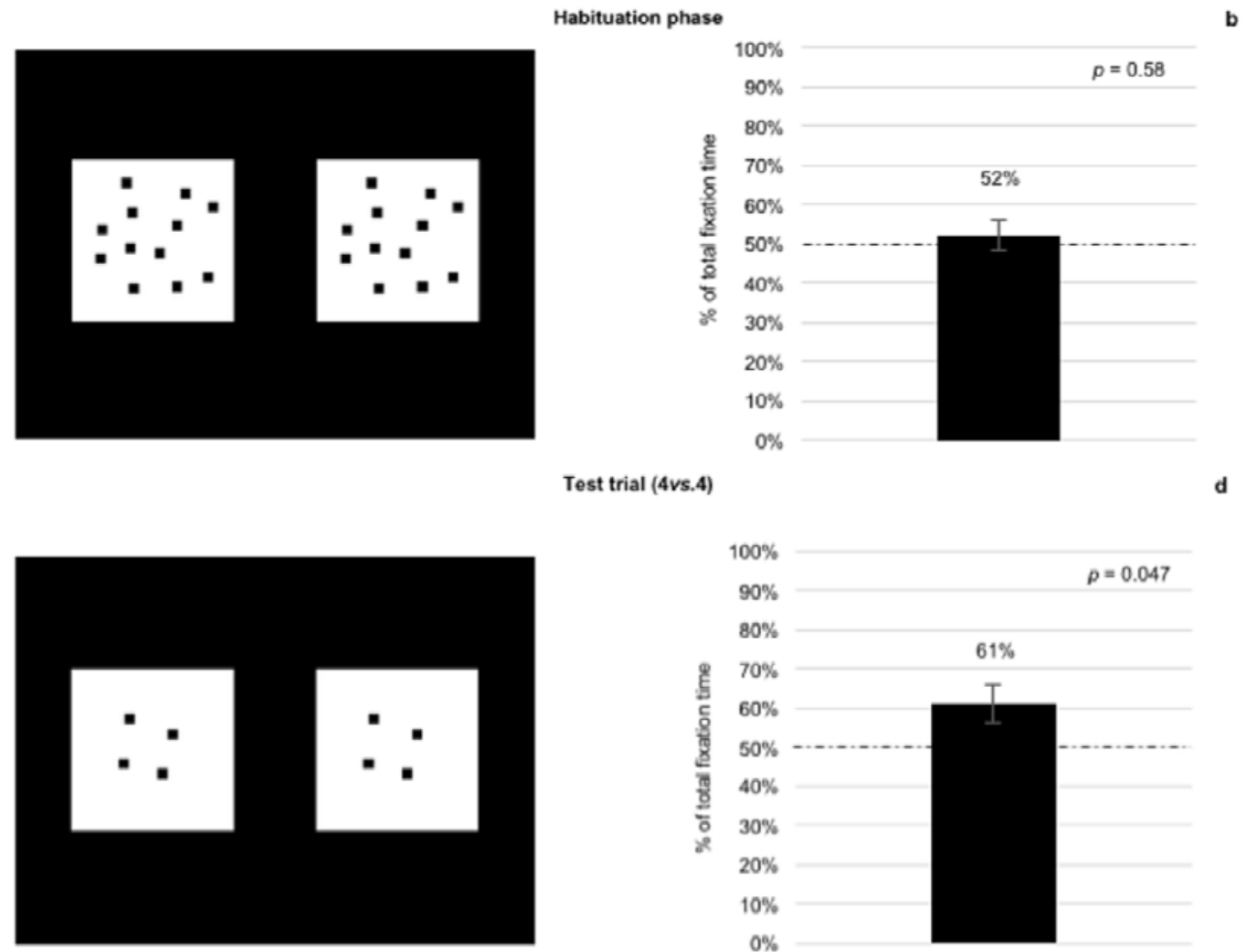

Test trial (4vs.4)

$\mathbf{e}$

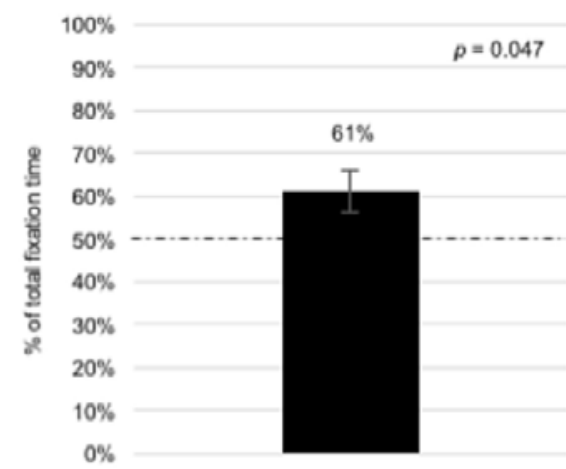

Test trial (36vs.36)
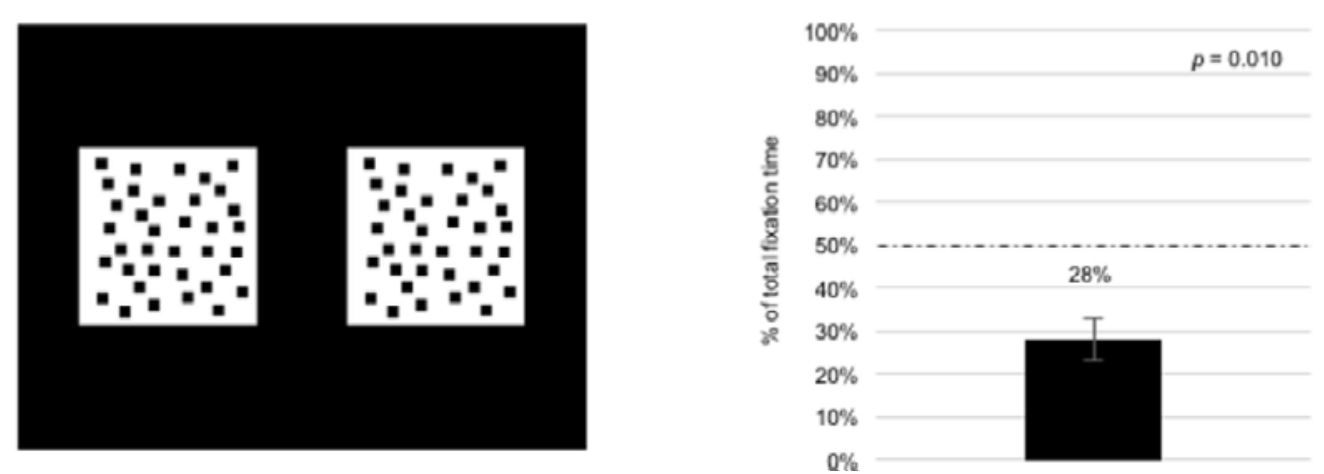

2 Figure 1. Stimuli and data on newborns' visual preference in Experiment 1.

3 Newborns were habituated with two identical stimuli, depicting 12 black squares of the same

4 dimension (a). Once they reached the habituation criterion, newborns underwent two test trials: one

5 with a small number (4vs.4) (c), and the other with a large number (36vs.36) (e). During

6 habituation, when the two stimuli depicted 12 squares, percentages of looking time toward the 
1 stimuli located on the left and on the right side of the screen did not significantly differ (b). In the

2 test trial, when the two stimuli depicted 4 squares (4vs.4), newborns looked longer at the left-

3 stimulus (d), when the stimuli depicted 36 squares (36vs.36), newborns looked longer at the right-

4 stimulus (f). Errors bars are standard error and dashed lines indicate chance level (50\%).

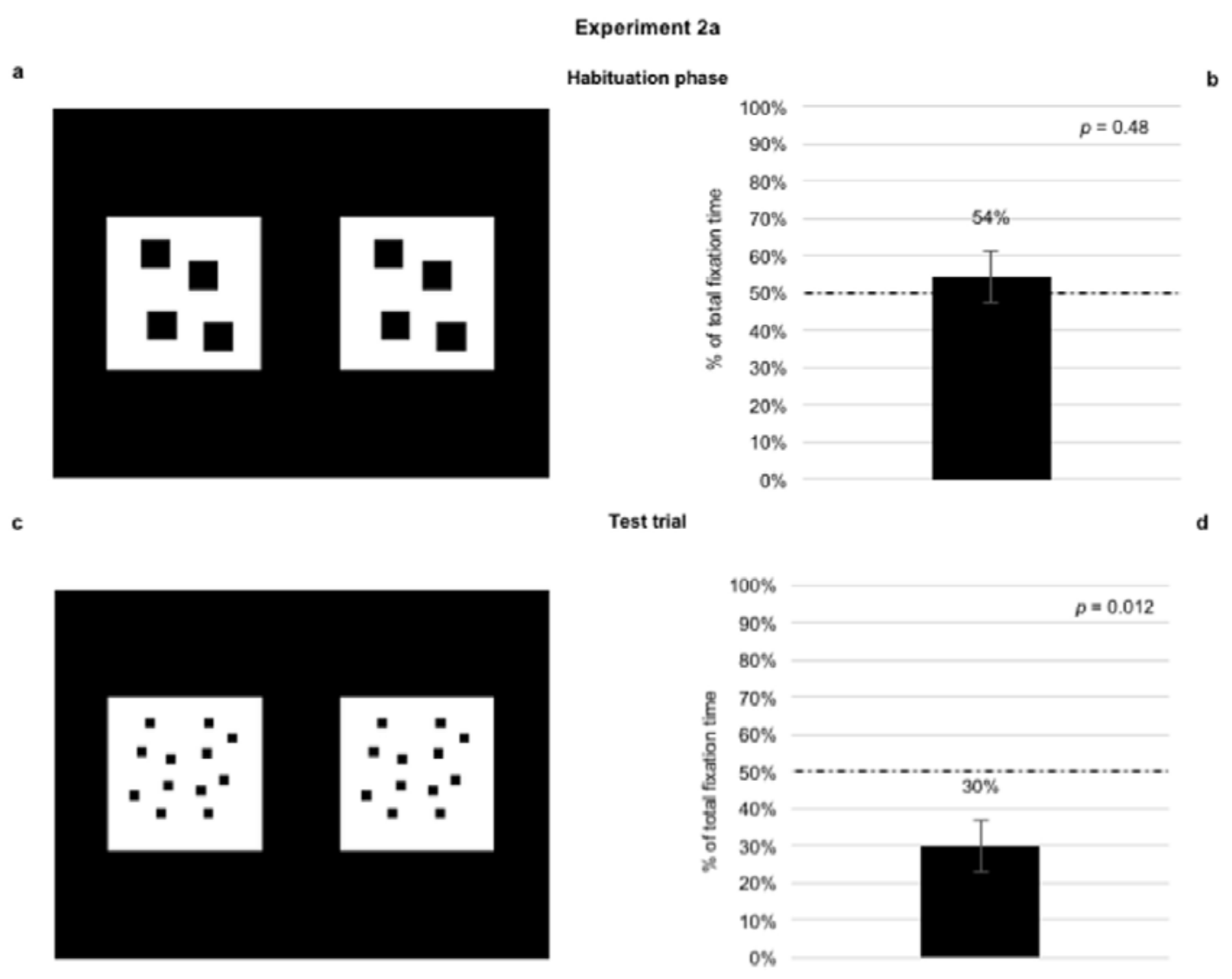


2 Figure 2. Stimuli and data on newborns' visual preference in Experiment 2a.

3 In Experiment 2a we simultaneously controlled for the overall perimeter and area. We habituated a

4 group of neonates with the number 4 (a) and then they were presented with the number 12 (12vs.12)

5 in the test trial (c). As for the habituation phase, newborns did not show any visual preference (b).

6 In the test trial, neonates looked longer at the right-stimulus (d). Error bars are standard error and

7 dashed lines indicate chance level (50\%).

a

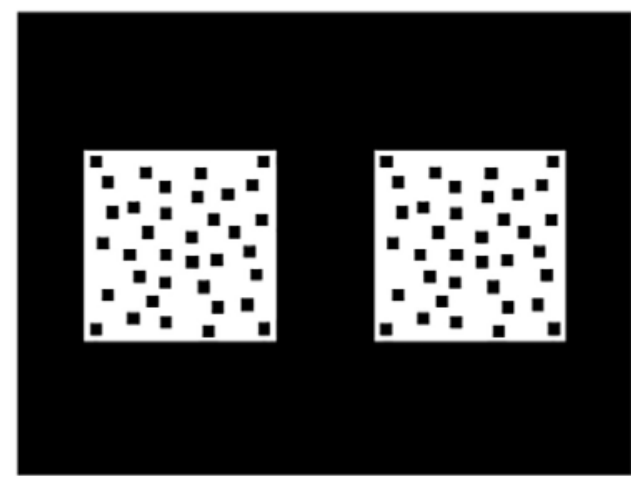

c

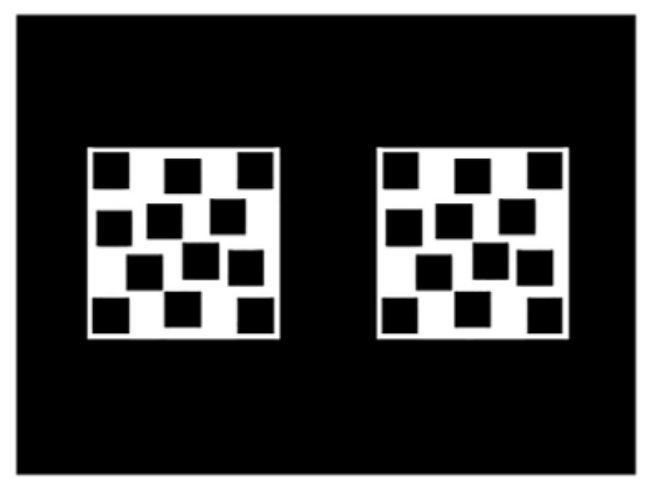

\section{Experiment 2b}

Habituation phase

$100 \%$

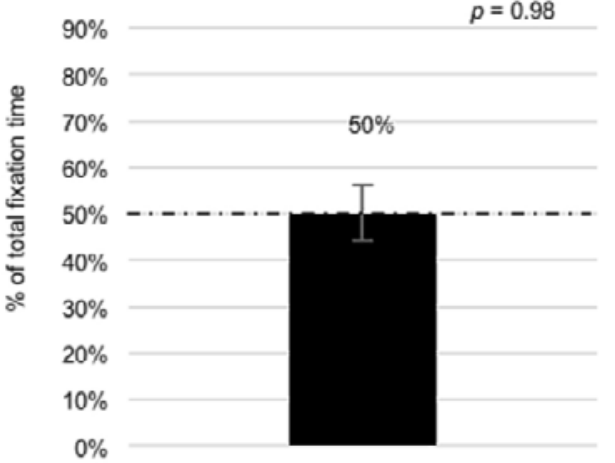

Test trial

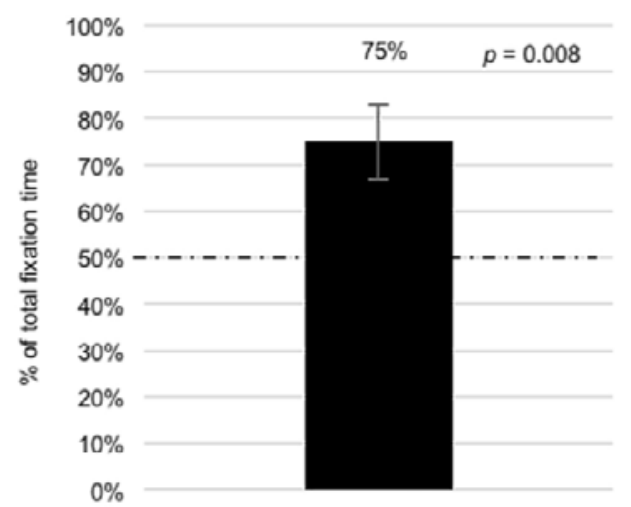

8

10 Figure 3. Stimuli and data on newborns' visual preference in Experiment 2b. 
1 In Experiment 2b, as in Experiment 2a, we simultaneously controlled for the overall perimeter and

2 area. We habituated a group of neonates with the number 36 (a) and then they were presented with

3 the number 12 (12vs.12) in the test trial (c). As for the habituation phase, newborns did not show

4 any visual preference (b). In the test trial, neonates looked longer at the left-stimulus (d). Error bars

5 are standard error and dashed lines indicate chance level (50\%). 


\section{Methods}

Participants. Sixty-nine (39 males) healthy, full-term, Caucasian newborns were selected

3 from the maternity ward of the Pediatric Clinic of the University of Padova. Data from twenty-one

4 babies ( 8 males) were discarded because i) they changed their state during testing $(n=10)$, ii) they

5 showed a positional bias $(\mathrm{n}=8)$, i.e. they looked in one direction for more than $80 \%$ of the time, 6 and, iii) they were considered outliers $(n=3)$.

The final sample consisted of forty-eight (31 males) newborns randomly assigned to one of

8 the three experimental conditions: twenty-four (10 males) took part in Experiment 1, twelve (6

9 males) in Experiment 2a and twelve (8 males) in Experiment 2b. All participants met the normal de-

10 livery screening criteria, had a birth weight between $2110 \mathrm{~g}$ and $4400 \mathrm{~g}(\mathrm{M}=3232.08 \mathrm{~g}, \mathrm{SD}=$

11 512.38), and an Apgar score of 9 at 5 min. Their mean postnatal age was 54.92 hours $(\mathrm{SD}=32.26$;

12 range: from 17 to 135 hours). All newborns were tested only if awake and in an alert state ${ }^{28}$, and af-

13 ter the parents had provided informed consent. All experimental procedures were licensed by the

14 Paediatric Clinic of the University of Padova (Protocol number 19147).

Apparatus and procedures. We conducted three experiments employing the infant-control

17 habituation procedure ${ }^{29,30}$. Stimuli presentation and data collection were performed using E-Prime $18 \quad 2.0$.

19 The baby sat on an experimenter's lap and white curtains were drawn on both sides of the newborn

20 to prevent interference from irrelevant distractors. The experimenter holding the baby was naive as

21 to the hypothesis being tested and to the stimuli being presented and was instructed to fix her/his

22 gaze on a monitor throughout the experimental session. Above the computer screen, the video

23 camera recorded the eye movements of the newborns to control their looking behavior on-line and 24 to allow off-line coding of their fixations. 
At the beginning of each experiment, a red disc on a black background appeared to attract

2 the newborn's gaze to the center of the monitor. In a continuous fashion, the disc changed in size

3 from small $(1.8 \mathrm{~cm})$ to large $(2.5 \mathrm{~cm})$ until the newborn's gaze was properly aligned with the red

4 disc. The red disc blinked at a rate of $300 \mathrm{~ms}$ on and $300 \mathrm{~ms}$ off. The sequence of trials was then

5 started by a second experimenter who watched the newborn's eyes through the monitor. When the

6 newborn's gaze was aligned with the red disk, the second experimenter pressed a keyboard key that

7 automatically turned off the central disc and activated the onset of the stimuli, thereby initiating the

8 sequence of trials.

The paradigm comprises the habituation phase and the test trials, in which two identical

10 stimuli were presented side-by-side. In the habituation phase, stimuli remained on the screen until

11 the habituation criterion was reached. Newborns were judged to have habituated when, from the

12 fourth fixation onward, the sum of any three consecutive fixations was $50 \%$ or less of the total of

13 the first three fixations ${ }^{31}$. A bilateral, rather than a central, presentation was selected for two rea-

14 sons: i) when newborns look at a centrally presented stimulus, it is difficult for a coder to decide

15 whether they are actually looking at the stimulus or simply not moving their eyes from the central

16 position; ii) at birth, the photoreceptors in the central fovea are very immature, resulting in poor vi-

17 sion in the central area of the visual field ${ }^{32,33}$.

18 During the test trials, one of the experimenters video recorded the newborns' visual behavior on

19 each stimulus. A test trial ended when newborns did not fixate on the display for at least $10 \mathrm{~s}$.

21 The stimuli were displayed on an Apple LED Cinema Display (Flat Panel 30") computer monitor

22 (refresh rate $=60 \mathrm{~Hz}$, resolution $2560 \times 1600$ pixels). 
Data Scoring and Statistical Analyses. Two coders, unaware of the stimuli presented,

2 analyzed off-line, the videos by coding newborn's eye movements frame by- frame.

3 The dependent variables that we measured, as in previous studies ${ }^{34,35}$, were some of those suggested

4 by Cohen's model of attention ${ }^{36}$ as indexes of the attention-getting and of the attention-holding

5 mechanisms. As indexes of attention-getting mechanisms, the coders recorded separately for each

6 stimulus and each position, the direction of the first fixation and the number of orientations for the

7 two stimuli. This latter measure was then converted to a percentage score by computing the total

8 number of orientations towards a given stimulus divided by the total number of orientations towards

9 both stimuli in each visual preference condition, X 100. As indexes of attention-holding mecha-

10 nisms, the coders recorded the longest fixation and the total fixation time (i.e., the sum of all fixa-

11 tions) towards the two stimuli. Also in the case of the total fixation time, we calculated the percent-

12 age of visual preference, that is, the length of time for which each newborn looked at a given stimu-

13 lus divided by the total time spent looking at both stimuli in each test trial, X 100 .

14 The mean estimated reliability between observers on the $64.58 \%$ of the overall participants (i.e., 31

15 out of 48 newborns) was Pearson's $r=0.93, \mathrm{p}<0.001$.

16 Data of Experiment 1 were analyzed as follow:

17 i) a first analysis was conducted on the percentage of total fixation time towards the

18 left position during the habituation phase, to control for any spontaneous a-priori

$19 \quad$ preference for a specific position (i.e., spatial biases);

20 i) a repeated measure ANOVA with Test Trials Order of stimuli presentation (4-36 and

21 36-4) as a between-participants factor and Stimulus (4vs.4 versus 36vs.36) as within-

22 participants factor on the percentage of total fixation time and number of orientations

$23 \quad$ toward left stimuli was carried out; 
ii) a two-sided one sample t-test on percentage of the number of orientations and a binomial test on the direction of the first fixation in the first test trial;

iii) a two-sided one sample t-test on percentage of total fixation time and a two-sided paired t-test (left vs. right) on the duration of the longest fixation in the first test trial.

In Experiment $2 \mathrm{a}$ and $2 \mathrm{~b}$, we run the following analyses:

i) we replicated the same analysis carried out in Experiment 1 during the habituation phase to control for any spontaneous a-priori preference for a specific position (i.e., spatial biases);

i) a univariate ANOVA with Experiment (2a and 2b) as a between-participants factor on the percentage of total fixation time and number of orientations toward left stimuli was carried out, in order to test relativity of SNA;

ii) a two-sided one sample t-test on percentage of the number of orientations and a binomial test on the direction of the first fixation were carried out;

iii) a two-sided one sample t-test on percentage of total fixation time and a two-sided paired t-test (left vs. right) on the duration of the longest fixation, were carried out.

Data were analyzed using SPSS software.

For a complete description of results, see SI.

\section{Data availability}

The datasets are available from the corresponding author on reasonable request. 